\title{
Some strong limit theorems for arrays of rowwise negatively orthant-dependent random variables
}

Aiting Shen

Correspondence: baret@sohu.com School of Mathematical Science, Anhui University, Hefei 230039, China

\begin{abstract}
In this article, the strong limit theorems for arrays of rowwise negatively orthantdependent random variables are studied. Some sufficient conditions for strong law of large numbers for an array of rowwise negatively orthant-dependent random variables without assumptions of identical distribution and stochastic domination are presented. As an application, the Chung-type strong law of large numbers for arrays of rowwise negatively orthant-dependent random variables is obtained.
\end{abstract}

MR(2000) Subject Classification: 60F15

Keywords: negatively orthant-dependent sequence, array of rowwise negatively orthant-dependent random variables, strong law of large numbers

\section{Introduction}

Let $\left\{X_{n}, n \geq 1\right\}$ be a sequence of random variables defined on a fixed probability space $(\Omega, \mathcal{F}, P)$ with value in a real space $\mathbb{R}$. We say that the sequence $\left\{X_{n}, n \geq 1\right\}$ satisfies the strong law of large numbers if there exist some increasing sequence $\left\{a_{n}, n \geq 1\right\}$ and some sequence $\left\{c_{n}, n \geq 1\right\}$ such that

$$
\frac{1}{a_{n}} \sum_{i=1}^{n}\left(X_{i}-c_{i}\right) \rightarrow 0 \quad \text { a.s. as } n \rightarrow \infty \text {. }
$$

Many authors have extended the strong law of large numbers for sequences of random variables to the case of triangular array of random variables and arrays of rowwise random variables. For more details about the strong law of large numbers for triangular array of random variables and arrays of rowwise random variables, one can refer to Gut [1], and so forth. In the case of independence, $\mathrm{Hu}$ and Taylor [2] proved the following strong law of large numbers.

Theorem 1.1. Let $\left\{X_{n i}: 1 \leq i \leq n, n \geq 1\right\}$ be a triangular array of rowwise independent random variables. Let $\left\{a_{n}, n \geq 1\right\}$ be a sequence of positive real numbers such that $0<a_{n} \uparrow \infty$. Let $g(t)$ be a positive, even function such that $g(|t|) /|t|^{p}$ is an increasing function of $|t|$ and $g(|t|) /|t|^{p+1}$ is a decreasing function of $|t|$, respectively, that is,

$$
\frac{g(|t|)}{|t|^{p}} \uparrow, \quad \frac{g(|t|)}{|t|^{p+1}} \downarrow \quad \text { as }|t| \uparrow
$$

(c) 2011 Shen; licensee Springer. This is an Open Access article distributed under the terms of the Creative Commons Attribution License (http://creativecommons.org/licenses/by/2.0), which permits unrestricted use, distribution, and reproduction in any medium, provided the original work is properly cited. 
for some nonnegative integer $p$. If $p \geq 2$ and

$$
\begin{gathered}
E X_{n i}=0, \\
\sum_{n=1}^{\infty} \sum_{i=1}^{n} E \frac{g\left(\left|X_{n i}\right|\right)}{g\left(a_{n}\right)}<\infty, \\
\sum_{n=1}^{\infty}\left(\sum_{i=1}^{n} E\left(\frac{X_{n i}}{a_{n}}\right)^{2}\right)^{2 k}<\infty,
\end{gathered}
$$

where $k$ is a positive integer, then

$$
\frac{1}{a_{n}} \sum_{i=1}^{n} X_{n i} \rightarrow 0 \quad \text { a.s. }
$$

In this article, we will consider the strong law of large numbers for arrays of rowwise negatively associated (NA) random variables. A finite collection of random variables $X_{1}, X_{2}, \ldots, X_{n}$ is said to be negatively orthant dependent (NOD) if

$$
P\left(X_{1}>x_{1}, X_{2}>x_{2}, \ldots, X_{n}>x_{n}\right) \leq \prod_{i=1}^{n} P\left(X_{i}>x_{i}\right)
$$

and

$$
P\left(X_{1} \leq x_{1}, X_{2} \leq x_{2}, \ldots, X_{n} \leq x_{n}\right) \leq \prod_{i=1}^{n} P\left(X_{i} \leq x_{i}\right)
$$

for all $x_{1}, x_{2}, \ldots, x_{n} \in \mathbb{R}$. An infinite sequence $\left\{X_{n}, n \geq 1\right\}$ is said to be NOD if every finite subcollection is NOD.

An array of random variables $\left\{X_{n i}, i \geq 1, n \geq 1\right\}$ is called rowwise NOD random variables if for every $n \geq 1,\left\{X_{n i}, i \geq 1\right\}$ is a sequence of NOD random variables.

The concept of NOD sequence was introduced by Joag-Dev and Proschan [3]. Obviously, independent random variables are NOD. Joag-Dev and Proschan [3] pointed out that NA (one can refer to Joag-Dev and Proschan [3]) random variables are NOD. They also presented an example in which $X=\left(X_{1}, X_{2}, X_{3}, X_{4}\right)$ possesses NOD, but does not possess NA. So we can see that NOD is weaker than NA. A number of limit theorems for NOD random variables have been established by many authors. We refer to Volodin [4] for the Kolmogorov exponential inequality, Asadian et al. [5] for the Rosental's-type inequality, Amini et al. [6,7], Klesov et al. [8], and Li et al. [9] for almost sure convergence, Amini and Bozorgnia [10,11], Kuczmaszewska [12], Taylor et al. [13], Zarei and Jabbari [14] and Wu [15] for complete convergence, and so on.

The main purpose of this article is to study the strong limit theorems for arrays of rowwise NOD random variables. As an application, the Chung-type strong law of large numbers for arrays of rowwise NOD random variables is obtained. We will give some sufficient conditions for strong law of large numbers for an array of rowwise NOD random variables without assumptions of identical distribution and stochastic domination. The results presented in this article are obtained using the truncated method and the Rosental's-type inequality of NOD random variables.

The main results of this article are depending on the following lemmas:

Lemma 1.1 (cf. Bozorgnia et al. [16]). Let random variables $X_{1}, X_{2}, \ldots, X_{n}$ be NOD, $f_{1}, f_{2}, \ldots, f_{n}$ be all nondecreasing (or all nonincreasing) functions, then random variables $f_{1}\left(X_{1}\right), f_{2}\left(X_{2}\right), \ldots, f_{n}\left(X_{n}\right)$ are NOD. 
Lemma 1.2 (cf. Asadian et al. [5]). Let $p \geq 2$ and $\left\{X_{n}, n \geq 1\right\}$ be a sequence of NOD random variables with $E X_{n}=0$ and $E\left|X_{n}\right|^{p}<\infty$ for every $n \geq 1$. Then, there exists a positive constant $C=C(p)$ depending only on $p$ such that for every $n \geq 1$

$$
E\left|\sum_{i=1}^{n} X_{i}\right|^{p} \leq C\left\{\sum_{i=1}^{n} E\left|X_{i}\right|^{p}+\left(\sum_{i=1}^{n} E X_{i}^{2}\right)^{p / 2}\right\} .
$$

Throughout the article, let $I(A)$ be the indicator function of the set $A$. $C$ denotes a positive constant which may be different in various places.

\section{Main results}

In this section, we will give some sufficient conditions for strong law of large numbers for an array of rowwise NOD random variables without assumptions of identical distribution and stochastic domination. Our main results are as follows.

Theorem 2.1. Let $\left\{X_{n i}: i \geq 1, n \geq 1\right\}$ be an array of rowwise NOD random variables and $\left\{a_{n}, n \geq 1\right\}$ be a sequence of positive real numbers. Let $\left\{g_{n}(t), n \geq 1\right\}$ be a sequence of positive, even functions such that $g_{n}(|t|)$ is an increasing function of $|t|$ and $g_{n}(|t|) /|t|$ is a decreasing function of $|t|$ for every $n \geq 1$, respectively, that is

$$
g_{n}(|t|) \uparrow, \quad \frac{g_{n}(|t|)}{|t|} \downarrow \quad \text { as }|t| \uparrow .
$$

If

$$
\sum_{n=1}^{\infty} \sum_{i=1}^{n} \frac{E g_{n}\left(\left|X_{n i}\right|\right)}{g_{n}\left(a_{n}\right)}<\infty
$$

then for any $\varepsilon>0$,

$$
\sum_{n=1}^{\infty} P\left(\left|\frac{1}{a_{n}} \sum_{i=1}^{n} X_{n i}\right|>\varepsilon\right)<\infty .
$$

Proof. For fixed $n \geq 1$, define

$$
\begin{aligned}
& X_{i}^{(n)}=-a_{n} I\left(X_{n i}<-a_{n}\right)+X_{n i} I\left(\left|X_{n i}\right| \leq a_{n}\right)+a_{n} I\left(X_{n i}>a_{n}\right), \quad i \geq 1, \\
& T_{j}^{(n)}=\frac{1}{a_{n}} \sum_{i=1}^{j}\left(X_{i}^{(n)}-E X_{i}^{(n)}\right), \quad j=1,2, \ldots, n .
\end{aligned}
$$

By Lemma 1.1, we can see that for fixed $n \geq 1,\left\{X_{i}^{(n)}, i \geq 1\right\}$ is still a sequence of NOD random variables. It is easy to check that for any $\varepsilon>0$,

$$
\left(\left|\frac{1}{a_{n}} \sum_{i=1}^{n} X_{n i}\right|>\varepsilon\right) \subset\left(\max _{1 \leq i \leq n}\left|X_{n i}\right|>a_{n}\right) \cup\left(\left|\frac{1}{a_{n}} \sum_{i=1}^{n} X_{i}^{(n)}\right|>\varepsilon\right),
$$

which implies that

$$
\begin{aligned}
P\left(\left|\frac{1}{a_{n}} \sum_{i=1}^{n} X_{n i}\right|>\varepsilon\right) & \leq P\left(\max _{1 \leq i \leq n}\left|X_{n i}\right|>a_{n}\right)+P\left(\left|\frac{1}{a_{n}} \sum_{i=1}^{n} X_{i}^{(n)}\right|>\varepsilon\right) \\
& \leq \sum_{i=1}^{n} P\left(\left|X_{n i}\right|>a_{n}\right)+P\left(\left|T_{n}^{(n)}\right|>\varepsilon-\left|\frac{1}{a_{n}} \sum_{i=1}^{n} E X_{i}^{(n)}\right|\right) .
\end{aligned}
$$


First, we will show that

$$
\left|\frac{1}{a_{n}} \sum_{i=1}^{n} E X_{i}^{(n)}\right| \rightarrow 0 \quad \text { as } n \rightarrow \infty .
$$

Actually, by conditions $g_{n}(|t|) \uparrow, g_{n}(|t|) /|t| \downarrow$ as $|t| \uparrow$ and (2.1), we have that

$$
\begin{aligned}
\left|\frac{1}{a_{n}} \sum_{i=1}^{n} E X_{i}^{(n)}\right| & \leq \sum_{i=1}^{n} P\left(\left|X_{n i}\right|>a_{n}\right)+\frac{1}{a_{n}} \sum_{i=1}^{n} E\left|X_{n i}\right| I\left(\left|X_{n i}\right| \leq a_{n}\right) \\
& \leq \sum_{i=1}^{n} \frac{E g_{n}\left(\left|X_{n i}\right|\right)}{g_{n}\left(a_{n}\right)}+\sum_{i=1}^{n} \frac{E g_{n}\left(\left|X_{n i}\right|\right) I\left(\left|X_{n i}\right| \leq a_{n}\right)}{g_{n}\left(a_{n}\right)} \\
& \leq 2 \sum_{i=1}^{n} \frac{E g_{n}\left(\left|X_{n i}\right|\right)}{g_{n}\left(a_{n}\right)} \rightarrow 0 \quad \text { as } n \rightarrow \infty,
\end{aligned}
$$

which implies (2.4). It follows from (2.3) and (2.4) that for $n$ large enough,

$$
P\left(\left|\frac{1}{a_{n}} \sum_{i=1}^{n} X_{n i}\right|>\varepsilon\right) \leq \sum_{i=1}^{n} P\left(\left|X_{n i}\right|>a_{n}\right)+P\left(\left|T_{n}^{(n)}\right|>\frac{\varepsilon}{2}\right) .
$$

Hence, to prove (2.2), we only need to show that

$$
\sum_{n=1}^{\infty} \sum_{i=1}^{n} P\left(\left|X_{n i}\right|>a_{n}\right)<\infty
$$

and

$$
\sum_{n=1}^{\infty} P\left(\left|T_{n}^{(n)}\right|>\frac{\varepsilon}{2}\right)<\infty
$$

The conditions $g_{n}(|t|) \uparrow$ as $|t| \uparrow$ and (2.1) yield that

$$
\sum_{n=1}^{\infty} \sum_{i=1}^{n} P\left(\left|X_{n i}\right|>a_{n}\right) \leq \sum_{n=1}^{\infty} \sum_{i=1}^{n} \frac{E g_{n}\left(\left|X_{n i}\right|\right)}{g_{n}\left(a_{n}\right)}<\infty,
$$

which implies (2.5).

By Markov's inequality, Lemma 1.2 (for $p=2$ ), $g_{n}(|t|) \uparrow, g_{n}(|t|) /|t| \downarrow$ as $|t| \uparrow$ and (2.1), we can get that

$$
\begin{aligned}
\sum_{n=1}^{\infty} P\left(\left|T_{n}^{(n)}\right|>\frac{\varepsilon}{2}\right) & \leq C \sum_{n=1}^{\infty} E\left|T_{n}^{(n)}\right|^{2} \leq C \sum_{n=1}^{\infty} \frac{1}{a_{n}^{2}} \sum_{i=1}^{n} E\left|X_{i}^{(n)}\right|^{2} \\
& \leq C \sum_{n=1}^{\infty} \sum_{i=1}^{n} P\left(\left|X_{n i}\right|>a_{n}\right)+C \sum_{n=1}^{\infty} \sum_{i=1}^{n} \frac{E\left|X_{n i}\right|^{2} I\left(\left|X_{n i}\right| \leq a_{n}\right)}{a_{n}^{2}} \\
& \leq C \sum_{n=1}^{\infty} \sum_{i=1}^{n} \frac{\mid E g_{n}\left(\left|X_{n i}\right|\right)}{g_{n}\left(a_{n}\right)}+C \sum_{n=1}^{\infty} \sum_{i=1}^{n} \frac{E\left|X_{n i}\right|^{2} I\left(\left|X_{n i}\right| \leq a_{n}\right)}{a_{n}^{2}} \\
& \leq C \sum_{n=1}^{\infty} \sum_{i=1}^{n} \frac{E g_{n}\left(\left|X_{n i}\right|\right)}{g_{n}\left(a_{n}\right)}+C \sum_{n=1}^{\infty} \sum_{i=1}^{n} \frac{E g_{n}\left(\left|X_{n i}\right|\right) I\left(\left|X_{n i}\right| \leq a_{n}\right)}{g_{n}\left(a_{n}\right)} \\
& \leq C \sum_{n=1}^{\infty} \sum_{i=1}^{n} \frac{E g_{n}\left(\left|X_{n i}\right|\right)}{g_{n}\left(a_{n}\right)}<\infty,
\end{aligned}
$$


which implies (2.6). This completes the proof of the theorem.

Corollary 2.1. Under the conditions of Theorem 2.1,

$$
\frac{1}{a_{n}} \sum_{i=1}^{n} X_{n i} \rightarrow 0 \quad \text { a.s. }
$$

Theorem 2.2. Let $\left\{X_{n i}: i \geq 1, n \geq 1\right\}$ be an array of rowwise NOD random variables and $\left\{a_{n}, n \geq 1\right\}$ be a sequence of positive real numbers. Let $\left\{g_{n}(t), n \geq 1\right\}$ be a sequence of nonnegative, even functions such that $g_{n}(|t|)$ is an increasing function of $|t|$ for every $n \geq 1$. Assume that there exists a constant $\delta>0$ such that $g_{n}(t) \geq \delta$ for $0<t \leq 1$. If

$$
\sum_{n=1}^{\infty} \sum_{i=1}^{n} E g_{n}\left(\frac{X_{n i}}{a_{n}}\right)<\infty
$$

then for any $\varepsilon>0$, (2.2) holds true.

Proof. We use the same notations as that in Theorem 2.1. The proof is similar to that of Theorem 2.1 .

First, we will show that (2.4) holds true. In fact, by the conditions $g_{n}(t) \geq \delta t$ for $0<t$ $\leq 1$ and (2.7), we have that

$$
\begin{aligned}
\left|\frac{1}{a_{n}} \sum_{i=1}^{n} E X_{i}^{(n)}\right| & \leq \sum_{i=1}^{n} P\left(\left|X_{n i}\right|>a_{n}\right)+\sum_{i=1}^{n} E\left(\frac{\left|X_{n i}\right|}{a_{n}} I\left(\left|X_{n i}\right| \leq a_{n}\right)\right) \\
& \leq \frac{1}{\delta} \sum_{i=1}^{n} E g_{n}\left(\frac{X_{n i}}{a_{n}}\right)+\frac{1}{\delta} \sum_{i=1}^{n} E g_{n}\left(\frac{X_{n i}}{a_{n}}\right) I\left(\left|X_{n i}\right| \leq a_{n}\right) \\
& \leq \frac{2}{\delta} \sum_{i=1}^{n} E g_{n}\left(\frac{X_{n i}}{a_{n}}\right) \rightarrow 0 \text { as } n \rightarrow \infty,
\end{aligned}
$$

which implies (2.4).

According to the proof of Theorem 2.1, we only need to prove that (2.5) and (2.6) hold true.

When $\left|X_{n i}\right|>a_{n}>0$, we have $g_{n}\left(\frac{X_{n i}}{a_{n}}\right) \geq g_{n}(1) \geq \delta$, which yields that

$$
P\left(\left|X_{n i}\right|>a_{n}\right)=E I\left(\left|X_{n i}\right|>a_{n}\right) \leq \frac{1}{\delta} E g_{n}\left(\frac{X_{n i}}{a_{n}}\right) .
$$

Hence,

$$
\sum_{n=1}^{\infty} \sum_{i=1}^{n} P\left(\left|X_{n i}\right|>a_{n}\right) \leq \frac{1}{\delta} \sum_{n=1}^{\infty} \sum_{i=1}^{n} E g_{n}\left(\frac{X_{n i}}{a_{n}}\right)<\infty
$$

which implies (2.5).

By Markov's inequality, Lemma 1.2 (for $p=2$ ), $g_{n}(t) \geq \delta t$ for $0<t \leq 1$ and (2.7), we can get that 


$$
\begin{aligned}
\sum_{n=1}^{\infty} P\left(\left|T_{n}^{(n)}\right|>\frac{\varepsilon}{2}\right) & \leq C \sum_{n=1}^{\infty} \sum_{i=1}^{n} P\left(\left|X_{n i}\right|>a_{n}\right)+C \sum_{n=1}^{\infty} \sum_{i=1}^{n} \frac{E\left|X_{n i}\right|^{2} I\left(\left|X_{n i}\right| \leq a_{n}\right)}{a_{n}^{2}} \\
& \leq C+C \sum_{n=1}^{\infty} \sum_{i=1}^{n} \frac{E\left|X_{n i}\right| I\left(\left|X_{n i}\right| \leq a_{n}\right)}{a_{n}} \\
& \leq C+C \sum_{n=1}^{\infty} \sum_{i=1}^{n} E g_{n}\left(\frac{X_{n i}}{a_{n}}\right) I\left(\left|X_{n i}\right| \leq a_{n}\right) \\
& \leq C+C \sum_{n=1}^{\infty} \sum_{i=1}^{n} E g_{n}\left(\frac{X_{n i}}{a_{n}}\right)<\infty,
\end{aligned}
$$

which implies (2.6). This completes the proof of the theorem.

Corollary 2.2. Let $\left\{X_{n i}, i \geq 1, n \geq 1\right\}$ be an array of rowwise NOD random variables and $\left\{a_{n}, n \geq 1\right\}$ be a sequence of positive real numbers. If there exists a constant $\beta \in(0$, 1] such that

$$
\sum_{n=1}^{\infty} \sum_{i=1}^{n} E\left(\frac{\left|X_{n i}\right|^{\beta}}{\left|a_{n}\right|^{\beta}+\left|X_{n i}\right|^{\beta}}\right)<\infty,
$$

then (2.2) holds true.

Proof. In Theorem 2.2, we take

$$
g_{n}(t) \equiv \frac{|t|^{\beta}}{1+|t|^{\beta}}, \quad 0<\beta \leq 1, n \geq 1 .
$$

It is easy to check that $\left\{g_{n}(t), n \geq 1\right\}$ is a sequence of nonnegative, even functions such that $g_{n}(|t|)$ is an increasing function of $|t|$ for every $n \geq 1$. And

$$
g_{n}(t) \geq \frac{1}{2} t^{\beta} \geq \frac{1}{2} t, \quad 0<t \leq 1,0<\beta \leq 1 .
$$

Therefore, by Theorem 2.2, we can easily get (2.2).

Corollary 2.3. Under the conditions of Theorem 2.2 or Corollary 2.2,

$$
\frac{1}{a_{n}} \sum_{i=1}^{n} X_{n i} \rightarrow 0 \quad \text { a.s. }
$$

Theorem 2.3. Let $\left\{X_{n i}: i \geq 1, n \geq 1\right\}$ be an array of rowwise NOD random variables and $\left\{a_{n}, n \geq 1\right\}$ be a sequence of positive real numbers. $E X_{n i}=0, i \geq 1, n \geq 1$. Let $\left\{g_{n}\right.$ $(x), n \geq 1\}$ be a sequence of nonnegative, even functions. Assume that there exist $\beta \in(1$, 2] and $\delta>0$ such that $g_{n}(x) \geq \delta x^{\beta}$ for $0<x \leq 1$ and there exists a $\delta>0$ such that $g_{n}(x) \geq$ $\delta x$ for $x>1$. If (2.7) satisfies, then for any $\varepsilon>0$, (2.2) holds true.

Proof. We use the same notations as that in Theorem 2.1. The proof is similar to that of Theorem 2.1 .

First, we will show that (2.4) holds true. Actually, by the conditions $E X_{n i}=0, g_{n}(x) \geq$ $\delta x$ for $x>1$ and (2.7), we have that 


$$
\begin{aligned}
\left|\frac{1}{a_{n}} \sum_{i=1}^{n} E X_{i}^{(n)}\right| & \leq \sum_{i=1}^{n} P\left(\left|X_{n i}\right|>a_{n}\right)+\left|\frac{1}{a_{n}} \sum_{i=1}^{n} E X_{n i} I\left(\left|X_{n i}\right|>a_{n}\right)\right| \\
& \leq 2 \sum_{i=1}^{n} E\left(\frac{\left|X_{n i}\right|}{a_{n}} I\left(\left|X_{n i}\right|>a_{n}\right)\right) \\
& \leq \frac{2}{\delta} \sum_{i=1}^{n} E g_{n}\left(\frac{X_{n i}}{a_{n}}\right) I\left(\left|X_{n i}\right|>a_{n}\right) \\
& \leq \frac{2}{\delta} \sum_{i=1}^{n} E g_{n}\left(\frac{X_{n i}}{a_{n}}\right) \rightarrow 0 \text { as } n \rightarrow \infty
\end{aligned}
$$

which implies (2.4). Hence, to prove (2.2), we only need to show that (2.5) and (2.6) hold true.

The conditions $g_{n}(x) \geq \delta x$ for $x>1$ and (2.1) yield that

$$
\begin{aligned}
\sum_{n=1}^{\infty} \sum_{i=1}^{n} P\left(\left|X_{n i}\right|>a_{n}\right) & =\sum_{n=1}^{\infty} \sum_{i=1}^{n} E I\left(\left|X_{n i}\right|>a_{n}\right) \\
& \leq \sum_{n=1}^{\infty} \sum_{i=1}^{n} E\left(\frac{\left|X_{n i}\right|}{a_{n}} I\left(\left|X_{n i}\right|>a_{n}\right)\right) \\
& \leq \frac{1}{\delta} \sum_{n=1}^{\infty} \sum_{i=1}^{n} E g_{n}\left(\frac{X_{n i}}{a_{n}}\right) I\left(\left|X_{n i}\right|>a_{n}\right) \\
& \leq \frac{1}{\delta} \sum_{n=1}^{\infty} \sum_{i=1}^{n} E g_{n}\left(\frac{X_{n i}}{a_{n}}\right)<\infty
\end{aligned}
$$

which implies (2.5).

By Markov's inequality, Lemma 1.2 (for $p=2$ ), $g_{n}(x) \geq \delta x^{\beta}$ for $1<\beta \leq 2,0<x \leq 1$ and (2.7), we can get that

$$
\begin{aligned}
\sum_{n=1}^{\infty} P\left(\left|T_{n}^{(n)}\right|>\frac{\varepsilon}{2}\right) & \leq C \sum_{n=1}^{\infty} \sum_{i=1}^{n} P\left(\left|X_{n i}\right|>a_{n}\right)+C \sum_{n=1}^{\infty} \sum_{i=1}^{n} \frac{E\left|X_{n i}\right|^{2} I\left(\left|X_{n i}\right| \leq a_{n}\right)}{a_{n}^{2}} \\
& \leq C+C \sum_{n=1}^{\infty} \sum_{i=1}^{n} \frac{E\left|X_{n i}\right|^{\beta} I\left(\left|X_{n i}\right| \leq a_{n}\right)}{a_{n}^{\beta}} \\
& \leq C+C \sum_{n=1}^{\infty} \sum_{i=1}^{n} E g_{n}\left(\frac{X_{n i}}{a_{n}}\right) I\left(\left|X_{n i}\right| \leq a_{n}\right) \\
& \leq C+C \sum_{n=1}^{\infty} \sum_{i=1}^{n} E g_{n}\left(\frac{X_{n i}}{a_{n}}\right)<\infty
\end{aligned}
$$

which implies (2.6). This completes the proof of the theorem. $\square$

Corollary 2.4. Let $\left\{X_{n i}, i \geq 1, n \geq 1\right\}$ be an array of rowwise NOD random variables and $\left\{a_{n}, n \geq 1\right\}$ be a sequence of positive real numbers. $E X_{n i}=0, i \geq 1, n \geq 1$. If there exists a constant $\beta \in(1,2]$ such that

$$
\sum_{n=1}^{\infty} \sum_{i=1}^{n} E\left(\frac{\left|X_{n i}\right|^{\beta}}{a_{n}\left|X_{n i}\right|^{\beta-1}+a_{n}^{\beta}}\right)<\infty,
$$

then (2.2) holds true. 
Proof. In Theorem 2.3, we take

$$
g_{n}(x) \equiv \frac{|x|^{\beta}}{1+|x|^{\beta-1}}, \quad 1<\beta \leq 2, n \geq 1 .
$$

It is easy to check that $\left\{g_{n}(x), n \geq 1\right\}$ is a sequence of nonnegative, even functions satisfying

$$
g_{n}(x) \geq \frac{1}{2} x^{\beta}, \quad 0<x \leq 1,1<\beta \leq 2 \quad \text { and } \quad g_{n}(x) \geq \frac{1}{2} x, \quad x>1 .
$$

Therefore, by Theorem 2.3, we can easily get (2.2).

Furthermore, by Corollaries 2.2 and 2.4, we can get the following important Chungtype strong law of large numbers for arrays of rowwise NOD random variables.

Corollary 2.5. Let $\left\{X_{n i}, i \geq 1, n \geq 1\right\}$ be an array of rowwise NOD random variables and $\left\{a_{n}, n \geq 1\right\}$ be a sequence of positive real numbers. If there exists some $\beta \in(0,2]$ such that

$$
\sum_{n=1}^{\infty} \sum_{i=1}^{n} \frac{E\left|X_{n i}\right|^{\beta}}{a_{n}^{\beta}}<\infty,
$$

and $E X_{n i}=0, i \geq 1, n \geq 1$ if $\beta \in(1,2]$, then (2.2) holds true and $\frac{1}{a_{n}} \sum_{i=1}^{n} X_{n i} \rightarrow 0$ a.s.. For $\beta \geq 2$, we have the following result.

Theorem 2.4. Let $\left\{X_{n i}: i \geq 1, n \geq 1\right\}$ be an array of rowwise NOD random variables and $\left\{a_{n}, n \geq 1\right\}$ be a sequence of positive real numbers. Let $\left\{g_{n}(x), n \geq 1\right\}$ be a sequence of nonnegative, even functions. Assume that there exists some $\beta \geq 2$ such that $g_{n}(x) \geq$ $\delta x^{\beta}$ for $x>0$. If

$$
\sum_{n=1}^{\infty} \sum_{i=1}^{n}\left[E g_{n}\left(\frac{X_{n i}}{a_{n}}\right)\right]^{1 / \beta}<\infty,
$$

then for any $\varepsilon>0$, (2.2) holds true.

Proof. We use the same notations as that in Theorem 2.1. The proof is similar to that of Theorem 2.1. It is easily seen that (2.8) implies that

$$
\sum_{n=1}^{\infty} \sum_{i=1}^{n} E g_{n}\left(\frac{X_{n i}}{M a_{n}}\right)<\infty
$$

and

$$
\sum_{n=1}^{\infty} \sum_{i=1}^{n}\left[E g_{n}\left(\frac{X_{n i}}{M a_{n}}\right)\right]^{2 / \beta}<\infty .
$$

First, we will show that (2.4) holds true. In fact, by Hölder's inequality, $g_{n}(x) \geq \delta x^{\beta}$ for $x>0,(2.8)$ and (2.9), we have that 


$$
\begin{aligned}
\left|\frac{1}{a_{n}} \sum_{i=1}^{n} E X_{i}^{(n)}\right| & \leq \sum_{i=1}^{n} P\left(\left|X_{n i}\right|>a_{n}\right)+\sum_{i=1}^{n} E\left(\frac{\left|X_{n i}\right|}{a_{n}} I\left(\left|X_{n i}\right| \leq a_{n}\right)\right) \\
& \leq \sum_{i=1}^{n} E\left(\frac{\left|X_{n i}\right|^{\beta}}{a_{n}^{\beta}} I\left(\left|X_{n i}\right|>a_{n}\right)\right)+\sum_{i=1}^{n}\left[E\left(\frac{\left|X_{n i}\right|^{\beta}}{a_{n}^{\beta}} I\left(\left|X_{n i}\right| \leq a_{n}\right)\right)\right]^{1 / \beta} \\
& \leq C \sum_{i=1}^{n} E g_{n}\left(\frac{X_{n i}}{a_{n}}\right)+C \sum_{i=1}^{n}\left[E g_{n}\left(\frac{X_{n i}}{a_{n}}\right) I\left(\left|X_{n i}\right| \leq a_{n}\right)\right]^{1 / \beta} \\
& \leq C \sum_{i=1}^{n} E g_{n}\left(\frac{X_{n i}}{a_{n}}\right)+C \sum_{i=1}^{n}\left[E g_{n}\left(\frac{X_{n i}}{a_{n}}\right)\right]^{1 / \beta} \rightarrow 0 \quad \text { as } n \rightarrow \infty,
\end{aligned}
$$

which implies (2.4). To prove (2.2), we only need to show that (2.5) and (2.6) hold true.

By the condition $g_{n}(x) \geq \delta x^{\beta}$ for $x>0$ again and (2.9), we have

$$
\begin{aligned}
\sum_{n=1}^{\infty} \sum_{i=1}^{n} P\left(\left|X_{n i}\right|>a_{n}\right) & =\sum_{n=1}^{\infty} \sum_{i=1}^{n} E I\left(\left|X_{n i}\right|>a_{n}\right) \\
& \leq \sum_{n=1}^{\infty} \sum_{i=1}^{n} E\left(\frac{\left|X_{n i}\right|^{\beta}}{a_{n}^{\beta}} I\left(\left|X_{n i}\right|>a_{n}\right)\right) \\
& \leq \frac{1}{\delta} \sum_{n=1}^{\infty} \sum_{i=1}^{n} E g_{n}\left(\frac{X_{n i}}{a_{n}}\right)<\infty,
\end{aligned}
$$

which implies (2.5).

By Markov's inequality, Lemma 1.2 (for $p=2$ ), $g_{n}(x) \geq \delta x^{\beta}$ for $x>0$ and (2.10), we can get that

$$
\begin{aligned}
\sum_{n=1}^{\infty} P\left(\left|T_{n}^{(n)}\right|>\frac{\varepsilon}{2}\right) & \leq C \sum_{n=1}^{\infty} \sum_{i=1}^{n} P\left(\left|X_{n i}\right|>a_{n}\right)+C \sum_{n=1}^{\infty} \sum_{i=1}^{n} \frac{E\left|X_{n i}\right|^{2} I\left(\left|X_{n i}\right| \leq a_{n}\right)}{a_{n}^{2}} \\
& \leq C+C \sum_{n=1}^{\infty} \sum_{i=1}^{n}\left[E\left(\frac{\left|X_{n i}\right|^{\beta}}{a_{n}^{\beta}} I\left(\left|X_{n i}\right| \leq a_{n}\right)\right)\right]^{2 / \beta} \\
& \leq C+C \sum_{n=1}^{\infty} \sum_{i=1}^{n}\left[E g_{n}\left(\frac{X_{n i}}{a_{n}}\right) I\left(\left|X_{n i}\right| \leq a_{n}\right)\right]^{2 / \beta} \\
& \leq C+C \sum_{n=1}^{\infty} \sum_{i=1}^{n}\left[E g_{n}\left(\frac{X_{n i}}{a_{n}}\right)\right]^{2 / \beta}<\infty,
\end{aligned}
$$

which implies (2.6). This completes the proof of the theorem. $\square$

\section{Acknowledgements}

The author was most grateful to the Editor Andrei Volodin and anonymous referees for careful reading of the manuscript and valuable suggestions which helped in improving an earlier version of this article.

Supported by the National Natural Science Foundation of China (11171001, 71071002) and the Academic Innovation Team of Anhui University (KJTD001B).

\section{Competing interests}

The authors declare that they have no competing interests.

Received: 18 April 2011 Accepted: 26 October 2011 Published: 26 October 2011

\section{References}

1. Gut, A: Complete convergence for arrays. Periodica Math Hungarica. 25(1):51-75 (1992). doi:10.1007/BF02454383 
2. Hu, TC, Taylor, RL: On the strong law for arrays and for the bootstrap mean and variance. Int J Math Math Sci. 20(2), 375-382 (1997). doi:10.1155/S0161171297000483

3. Joag-Dev, K, Proschan, F: Negative association of random variables with applications. Ann Stat. 11(1):286-295 (1983). doi:10.1214/aos/1176346079

4. Volodin, A: On the Kolmogorov exponential inequality for negatively dependent random variables. Pakistan J Stat. 18, 249-254 (2002)

5. Asadian, N, Fakoor, V, Bozorgnia, A: Rosental's type inequalities for negatively orthant dependent random variables. J Iranian Stat Soc. 5(1-2):66-75 (2006)

6. Amini, M, Azarnoosh, HA, Bozorgnia, A: The strong law of large numbers for negatively dependent generalized Gaussian random variables. Stochast Anal Appl. 22, 893-901 (2004)

7. Amini, $\mathrm{M}$, Zarei, H, Bozorgnia, A: Some strong limit theorems of weighted sums for negatively dependent generalized Gaussian random variables. Stat Probab Lett. 77, 1106-1110 (2007). doi:10.1016/j.spl.2007.01.015

8. Klesov, O, Rosalsky, A, Volodin, A: On the almost sure growth rate of sums of lower negatively dependent nonnegative random variables. Stat Probab Lett. 71, 193-202 (2005). doi:10.1016/j.spl.2004.10.027

9. Li, D, Rosalsky, A, Volodin, A: On the strong law of large numbers for sequences of pairwise negative quadrant dependent random variables. Bull Inst Math Acad Sin (New Ser). 1(2), 281-305 (2006)

10. Amini, M, Bozorgnia, A: Negatively dependent bounded random variable probability inequalities and the strong law of large numbers. J Appl Math Stochast Anal. 13, 261-267 (2000). doi:10.1155/S104895330000023X

11. Amini, $M$, Bozorgnia, A: Complete convergence for negatively dependent random variables. J Appl Math Stochast Anal. 16, 121-126 (2003). doi:10.1155/\$104895330300008X

12. Kuczmaszewska, A: On some conditions for complete convergence for arrays of rowwise negatively dependent random variables. Stochast Anal Appl. 24, 1083-1095 (2006). doi:10.1080/07362990600958754

13. Taylor, RL, Patterson, RF, Bozorgnia, A: A strong law of large numbers for arrays of rowwise negatively dependent random variables. Stochast Anal Appl. 20, 643-656 (2002). doi:10.1081/SAP-120004118

14. Zarei, H, Jabbari, H: Complete convergence of weighted sums under negative dependence. Stat Pap. (2009)

15. Wu, QY: Complete convergence for negatively dependent sequences of random variables. J Inequal Appl 2010, 10 (2010). Article ID 507293

16. Bozorgnia, A, Patterson, RF, Taylor, RL: Limit theorems for dependent random variables. World Congress Nonlinear Analysts'92. 1639-1650 (1996)

doi:10.1186/1029-242X-2011-93

Cite this article as: Shen: Some strong limit theorems for arrays of rowwise negatively orthant-dependent random variables. Journal of Inequalities and Applications 2011 2011:93.

\section{Submit your manuscript to a SpringerOpen ${ }^{\circ}$ journal and benefit from:}

- Convenient online submission

- Rigorous peer review

- Immediate publication on acceptance

- Open access: articles freely available online

- High visibility within the field

- Retaining the copyright to your article

Submit your next manuscript at $\gg$ springeropen.com 